\title{
Etude de l'influence du régime sur l'état histologique et physicochimique du glycogène chez Ascaris lumbricoides
}

\author{
par J. SAVEL et P. GEORGES \\ (Institut de Parasitologie - Faculté de Pharmacie, 4, av. de l'Observatoire - Paris-6")
}

\section{Résumé}

Les auteurs envisagent les conséquences du jeûne, chez Ascaris lumbricoides, sur les modifications que subit le glycogène tissulaire sur les plans histologique et physico-chimique.

L'étude histologique montre l'appauvrissement en glycogène de divers organes; dans l'ordre de déplétion décroissante: cellules musculaires, champs latéraux, gonades.

L'étude physico-chimique permet de distinguer, dans le bloc glycogénique, deux fractions de poids moléculaires différents. Pendant le jeûne, la plus légère s'appauvrit plus rapidement et plus considérablement que la plus lourde. En revanche, elle se reconstitue la première après renutrition par un glucide approprié.

\section{Summary}

The A.A. study, in an histological and physico-chemical point of view, the consequences of diet upon tissular glycogen in Ascaris lumbricoides.

Histologically, a fall of glycogen become obvious in different organs, i.e., in a decreasing order: muscular cells, lateral hypodermic cords and gonads.

By physico-chemical study, it appears that two fractions of non-equal molecular weight may be differenciated in the glycogenic block.

The lightest degenerates more quickly and more intensively during the diet than the heaviest; but, on the other hand, it first recovers when renutrition with a suitable glucid took place. 
On sait, depuis les travaux plus ou moins récents de nombreux auteurs, que le glycogène représente, chez l'Ascaris, le glucide quantitativement le plus important ( $3,5 \mathrm{~g}$ à $4 \mathrm{~g}$ pour $100 \mathrm{~g}$ en poids frais du parasite), principalement au niveau des cellules musculaires dans lesquelles a été trouvée une concentration en polysaccharide de l'ordre de 13 à $15 \mathrm{~g}$ pour $100 \mathrm{~g}$ d'organe frais $(1,3)$.

Il s'agit là, pour l'Ascaris, d'une réserve glucidique que l'animal est capable de mobiliser si les circonstances lui imposent un jeûne plus ou moins complet et qu'il peut ultérieurement reconstituer si un aliment convenable lui est fourni (1).

D'autre part, le glycogène ascaridien se présente, comme de nombreux glycogènes parasitaires, en un bloc hétérogène au sein duquel on peut observer deux fractions. Cette hétérogénéité a longtemps été fondée sur la distinction autrefois classique entre lyoglycogène ou glycogène libre et desmoglycogène associé avec des protéines pour former un complexe glucoprotéique.

Or, on sait aujourd'hui que cette distinction entre lyoglycogène et desmoglycogène est complètement périmée et il apparaît, d'autre part, à la lumière des travaux récents d'Orrell, Bueding et Reissig (8) que les divergences relevées dans les résultats publiés, les interprétations erronées qui ont été avancées sont facilement explicables par les incidences du procédé d'extraction sur les caractéristiques du polysaccharide obtenu ; c'est ainsi qu'on obtient des glycogènes de poids moléculaires très différents selon que l'on utilise un alcali fort comme la potasse, l'acide trichloracétique à froid ou l'eau bouillante.

Les auteurs précédents ont montré que l'extraction par l'eau froide en présence de certains solvants organiques était la mieux capable de fournir un produit dans l'état qui reflète le plus fidèlement celui sous lequel il existe dans l'organisme vivant.

Grâce à ce procédé, l'étude de la répartition du coefficient de sédimentation au cours de l'ultracentrifugation a permis de distinguer, dans le bloc glycogénique isolé, deux fractions de poids moléculaires différents, environ $45 \times 10^{7}$ pour la plus lourde et $50 \times 10^{6}$ pour la plus légère, les deux fractions étant quantitativement à peu près égales et correspondant à des images distinctes observées par la microscopie électronique [Harrap et Manners (4), Orrell et Bueding (6), Kjolbert, Manners et Wright (5), Orrell, Bueding et Reissig (8)].

Notre propos est d'étudier ici, par des observations histologiques, comment se répartit le glycogène de l'Ascaris selon les divers organes, de quelle manière le jeûne influence les différents dépôts suivant leur localisation, comment enfin évoluent les deux fractions du glycogène au cours du jeûne hydrocarboné et pendant la reconstitution des réserves, ce qui permettra peut être de préciser la signification physiologique de cette hétérogénéité au sein du bloc glycogénique.

\section{I. - ETUDE HISTOLOGIQUE DE LA REPARTITION DU GLYCOGENE}

\section{a) Chez l'Ascaris à régime glucidique normal.}

Cette étude a été effectuée après traitement des tissus de l'Ascaris par le fixateur de Carnoy, fixation d'ailleurs laborieuse qui demande un contact prolongé (dix jours environ) des fragments d'ascaris avec le réactif. L'inclusion se fait dans la paraffine 
et on pratique des coupes de $5 \mu$ d'épaisseur. Celles-ci sont colorées, soit par la méthode au P.A.S., soit au carmin de Best, certaines d'entre elles étant, au préalable, traitées par la salive afin de servir de préparations témoins débarrassées de leur glycogène.

Nous avons ainsi obtenu, après photomicrographie, des images dont nous donnons ici quelques exemples qui permettent de faire les constatations suivantes:

Cuticule: (Clichés $\left.\mathrm{n}^{\circ *} 1,3,4\right)$.

Nous n'y avons jamais observé la présence de glycogène.

HyPoderme et champs (Clichés $\left.\mathrm{n}^{\circ s} 1,3,4\right)$.

On note la présence de granules de glycogène tout au long de l'hypoderme sans que ceux-ci atteignent d'ailleurs une densité particulièrement remarquable.

Les champs dorsal et ventral présentent des taches plus ou moins étendues à côté de granules assez grossiers.

Les champs latéraux apparaissent comme l'une des plus importantes zones d'accumulation du glycogène. Si la lumière du canal est toujours vide de glycogène, le reste des deux champs en est toujours abondamment pourvu sous forme de masses rouges, plus ou moins volumineuses.

Cellules myo-épithéliales (Clichés $\left.\mathrm{n}^{\circ *} 1,3,4\right)$.

Elles contiennent d'importantes quantités de glycogène inégalement réparties dans le corps cellulaire. La partie contractile en est relativement pauvre alors que la partie renflée et la partie du bras qui y est adjacente en contiennent la majeure partie. Le glycogène y apparaît, soit en nappes, soit sous forme granulaire. Nous précisons que, déterminée par voie chimique, la concentration des cellules musculaires en glycogène est de 13 à $15 \mathrm{~g} \%$.

Il semble que les cellules musculaires de la région antérieure contiennent relativement moins de glycogène que celles de la partie postérieure.

TubE Digestif (Cliché $\mathrm{n}^{\circ} 2$ ).

Il est constitué d'une couche unique de cellules hautes et étroites limitées du côté lumière intestinale et du côté interne par deux bordures colorées en rouge vif par le P.A.S. Il s'agit de muco-polysaccharides résistant à l'action de l'amylase. En revanche, le cytoplasme cellulaire est parfois parsemé de micro-granules, eux aussi P.A.S.-positifs, mais qui disparaissent après action de l'amylase. Il ne semble pas que ces fines inclusions représentent un élément permanent de la cellule intestinale. Ces observations sont à rapprocher du fait que la teneur en glycogène de l'intestin, de l'ordre de $0,5 \mathrm{~g} \%$, est la plus faible trouvée dans les différents tissus de l'Ascaris,

Organes génitaux (Clichés $\left.\mathrm{n}^{\circ *} 5,6\right)$.

Les organes génitaux mâles sont pauvres en glycogène (chimiquement, 0,35 à $0,40 \mathrm{~g}$ pour $100 \mathrm{~g}$ ): quelques granules épais dans les éléments observables au long du tube testiculaire. 
Les organes génitaux femelles présentent des granules P.A.S.-positifs, perdant leur colorabilité après digestion par l'amylase. Ces granules sont surtout nombreux dans la partie ovarienne et deviennent de moins en moins denses au fur et à mesure que l'on se rapproche de l'extrémité distale du tractus génital, ce qui correspond aux données chimiques qui attribuent aux ovaires une teneur en glycogène supérieure $(6$ à $7 \mathrm{~g} \%)$ à celle des œufs situés dans les deux utérus, prêts à être pondus ( 2 à $3 \mathrm{~g}$ pour $100 \mathrm{~g}$ ).

\section{b) Chez l'Ascaris soumis au jeûne glucidique.}

Nous avons maintenu des ascaris, pendant cinq jours, en survie aseptique dans un milieu de survie convenablement équilibré mais dépourvu de tout glucide et nous avons observé pendant cette période une déplétion rapide en glycogène, de l'ordre de 40 à $50 \%$ par 24 heures, dans les deux premiers jours. Cet appauvrissement devient considérable, de 80 à $90 \%$ de la valeur initiale au bout de quatre à cinq jours et est fatal aux animaux si ceux-ci ne peuvent reconstituer leurs réserves glycogéniques à partir d'un glucide approprié.

Sur le plan histologique, cette déplétion glycogénique provoque les modifications suivantes, observées sur des coupes provenant d'animaux ayant subi un jeûne de 72 heures (Clichés $\mathrm{n}^{\circ \mathrm{s}} 7,8$ ).

Nous observons que le glycogène a complètement disparu des cellules musculaires dont la partie, auparavant dilatée, apparaît recroquevillée.

A noter également la diminution considérable du polysaccharide au niveau des champs. C'est dans les gonades que le glycogène paraît avoir été relativement le plus épargné, constatation confirmée par les déterminations chimiques effectuées pendant la même période, qui accusaient, pour le muscle, une perte de glycogène de $68 \%$, et pour l'ovaire de $45 \%$ seulement.

\section{II. - INFLUENCE DE LA DEPLETION ET DE LA RECUPERATION GLYCOGENIQUES SUR L'ETAT PHYSICO-CHIMIQUE DU GLYCOGENE}

Nous avons évoqué plus haut les travaux d'Orrell et coll. (8) où ces auteurs, grâce à une méthode d'extraction respectant l'état physico-chimique sous lequel se présente le glycogène dans le muscle, ont pu montrer que le polysaccharide se présente en deux fractions de poids moléculaires différents. De notre côté, nous avons suivi le comportement de chacune de ces fractions au cours de la déplétion glycogénique provoquée par le jeûne et pendant la phase de récupération assurée par l'apport d'un glucide bien choisi, en l'espèce le glucose.

Nous avons donc extrait, par la technique à l'eau froide décrite ci-après en annexe, le glycogène des cellules musculaires d'ascaris :

- retirés de l'intestin de porcs, juste après l'abattage ;

— au bout d'une période de 24 h de jeûne ; 


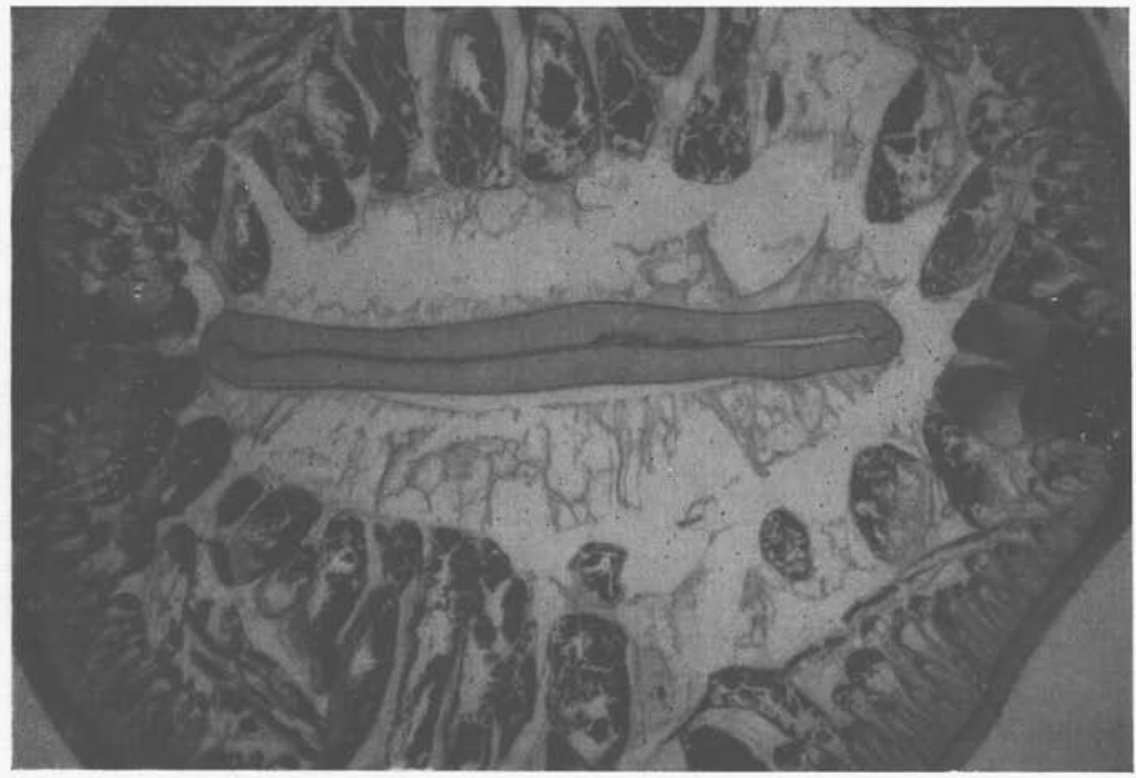

Fig. 1. - Coupe transversale

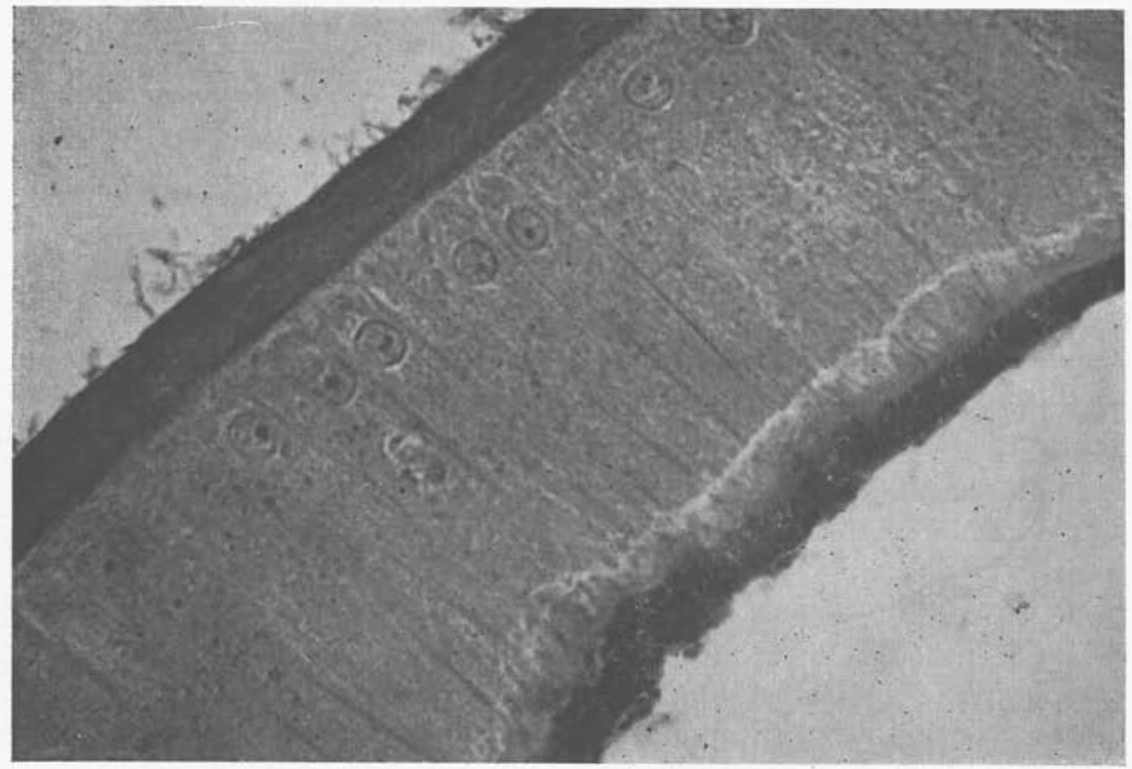

FIG. 2. - Intestin (coupe transversale)

Annales de Parasitologie humaine et comparée (Paris), t. 44, 1969, $\mathrm{n}^{\circ} 1$ 


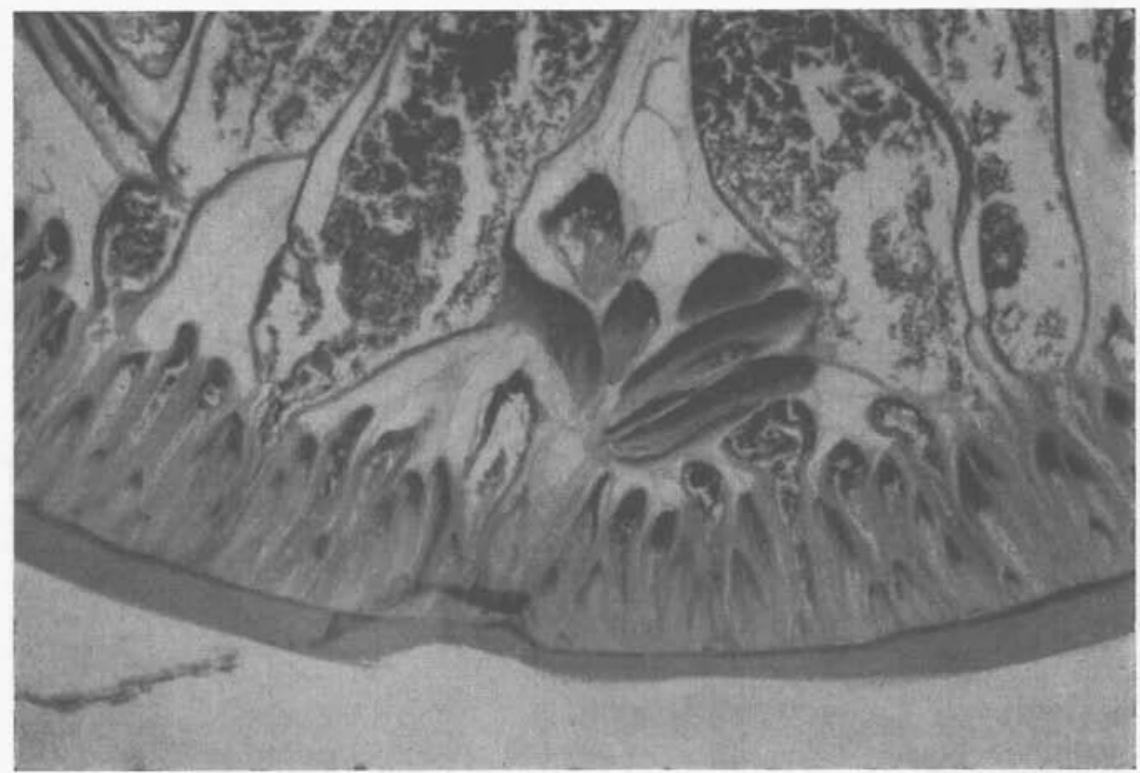

Fig. 3. - Myoblastes et champ dorsal

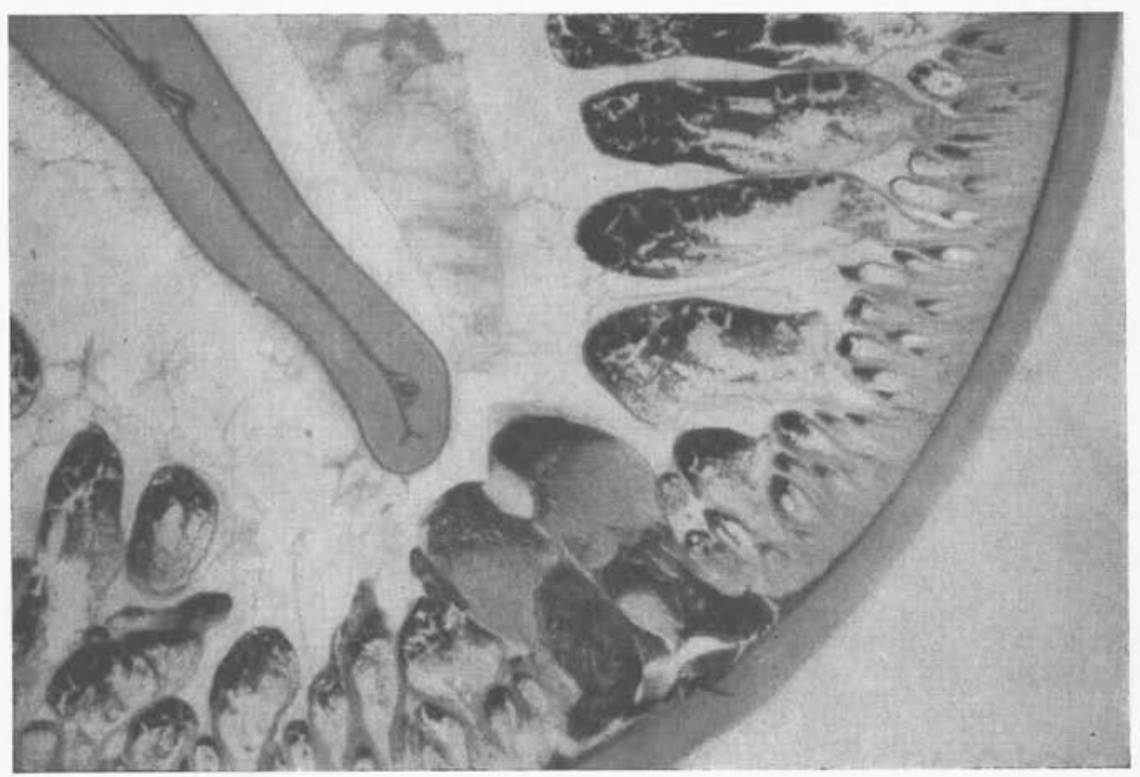

Fig. 4. - Myoblastes et champ latéral 


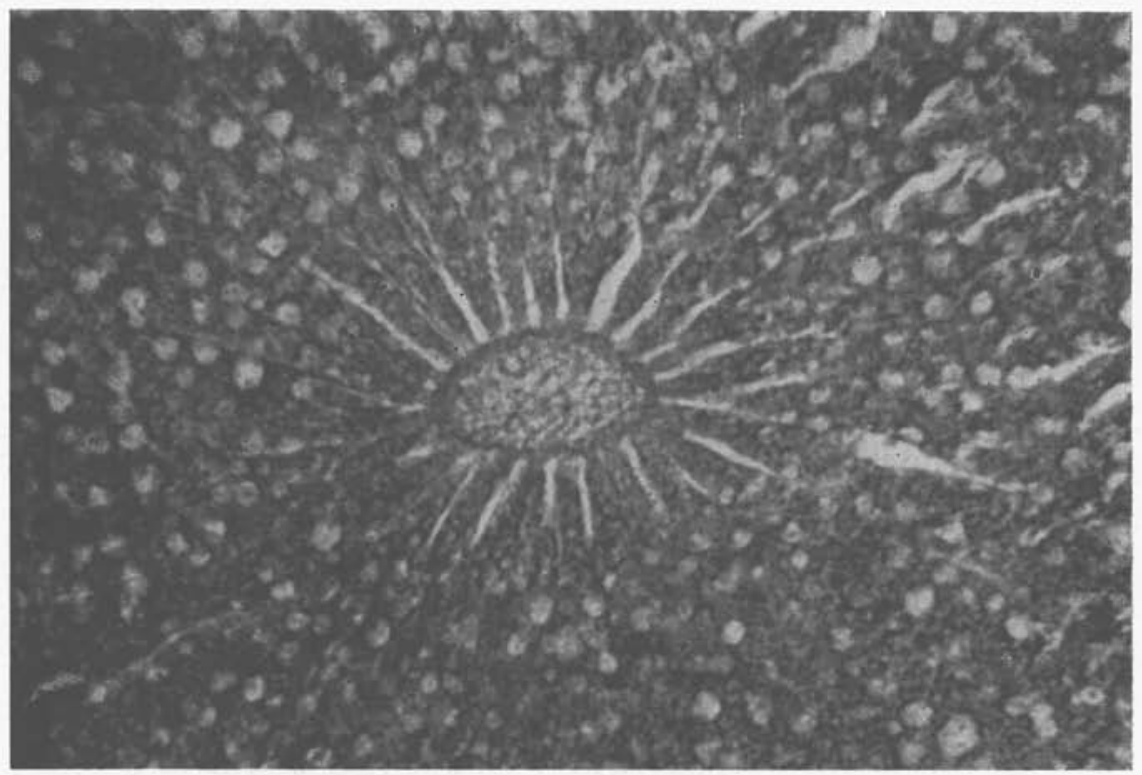

FIG. 5. - Ovaire (coupe transversale)

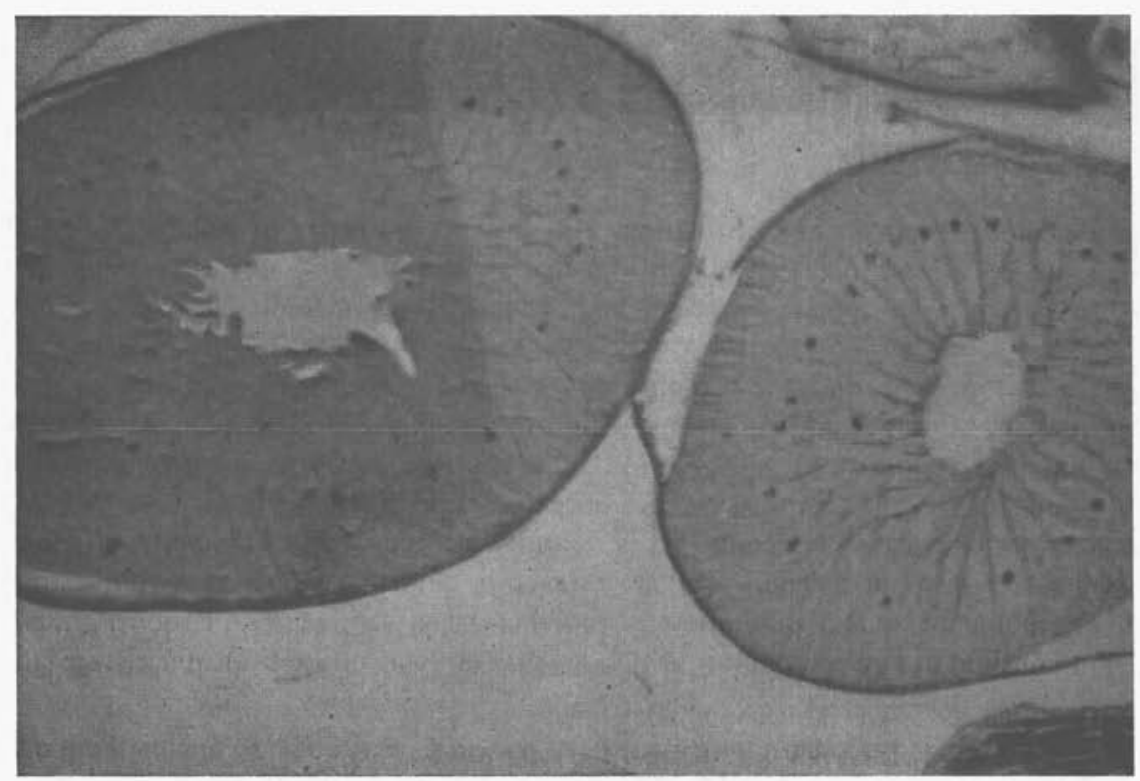

Fig. 6. - Ovaires avant et après contact de la salive 


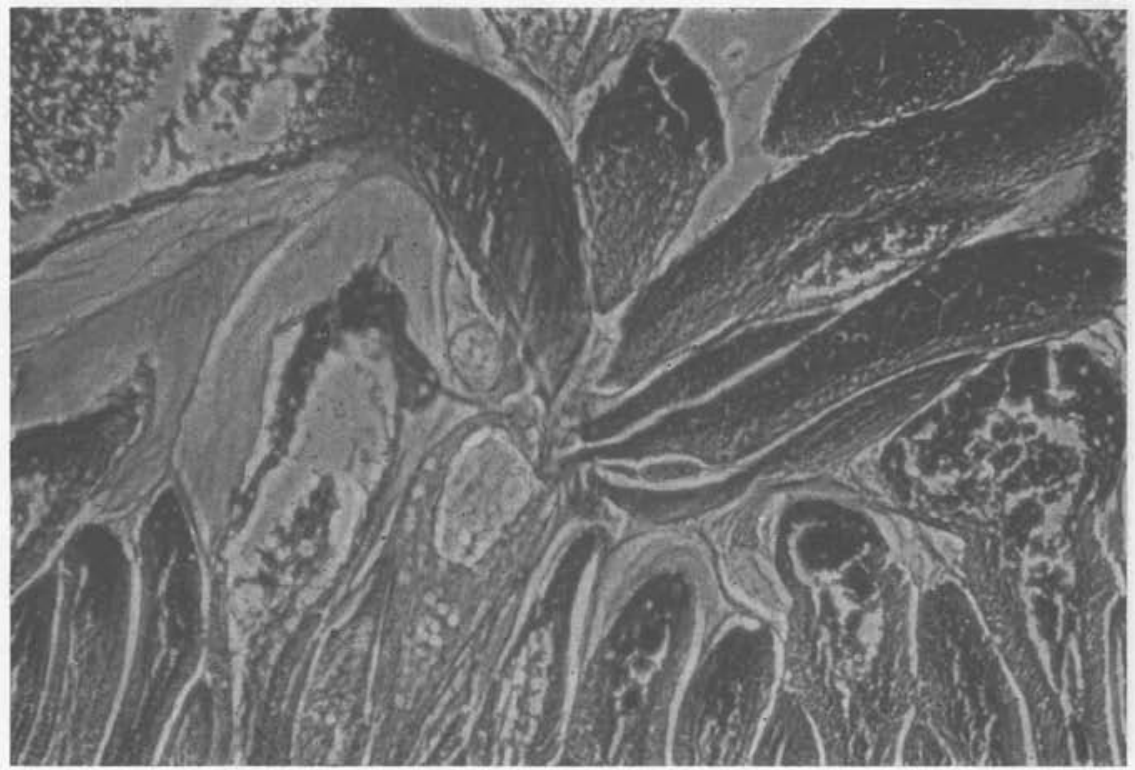

Fig. 7. - Myoblastes et champ dorsal avant le jeûne (contraste de phase)

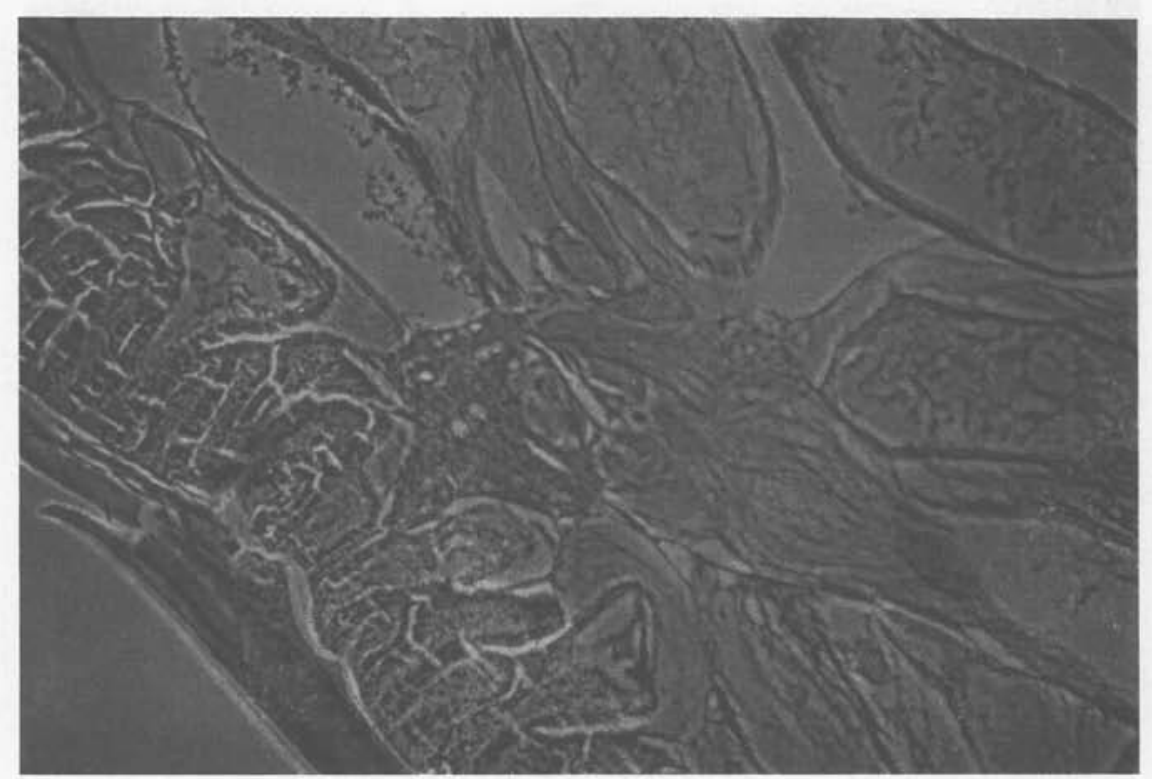

Fig. 8. - Myoblastes et champ dorsal après le jeûne (contraste de phase) - 
- au bout d'une période de 24 h de jeûne suivie d'une période de réalimentation glucosée de 24 heures.

La répartition du coefficient de sédimentation a été déterminée à l'aide d'une ultracentrifugeuse analytique Spinco type E, à raison de 9.341 tours par minute pendant 19 minutes.

Nous groupons dans le tableau $\mathrm{n}^{\circ} 1$ et sur les courbes de la figure $\mathrm{n}^{\circ} \mathrm{l}$ les résultats concernant la concentration du muscle en glycogène avant le jeûne $(A)$, après $24 \mathrm{~h}$ de jeûne (B) et après $24 \mathrm{~h}$ de restauration glycogénique $(C)$ dans un milieu de sur-

\section{TABLEAU I}

Comportement des deux fractions glycogéniques en fonction de la concentration du muscle en glycogène chez Ascaris lumbricoides

\begin{tabular}{|c|c|c|c|c|c|c|c|}
\hline & \multirow{3}{*}{$\begin{array}{c}\text { Glycogène } \\
\mathrm{g} \%\end{array}$} & \multicolumn{2}{|c|}{$\%$} & \multicolumn{4}{|c|}{ Coefficient de Sédimentation } \\
\hline & & \multirow{2}{*}{$\begin{array}{l}\text { fraction } \\
\text { légère }\end{array}$} & \multirow{2}{*}{$\begin{array}{l}\text { fraction } \\
\text { lourde }\end{array}$} & \multicolumn{2}{|c|}{ Fraction légère } & \multicolumn{2}{|c|}{ Fraction lourde } \\
\hline & & & & limites & moyenne & limites & moyenne \\
\hline $\begin{array}{c}\text { Jeûne } \\
0 \mathrm{~h}\end{array}$ & 14,7 & 49,6 & 50,4 & $20-525$ & 180 & $525-1000$ & 1060 \\
\hline $\begin{array}{c}24 \mathrm{~h} \\
\text { Renutri- } \\
\text { tion } \\
\text { glucosée }\end{array}$ & 8 & 39 & 61 & $20-325$ & 100 & $315-1500$ & 880 \\
\hline $24 \mathrm{~h}$ & 10,4 & 47 & 53 & $20-500$ & 180 & $500-1850$ & 1100 \\
\hline
\end{tabular}

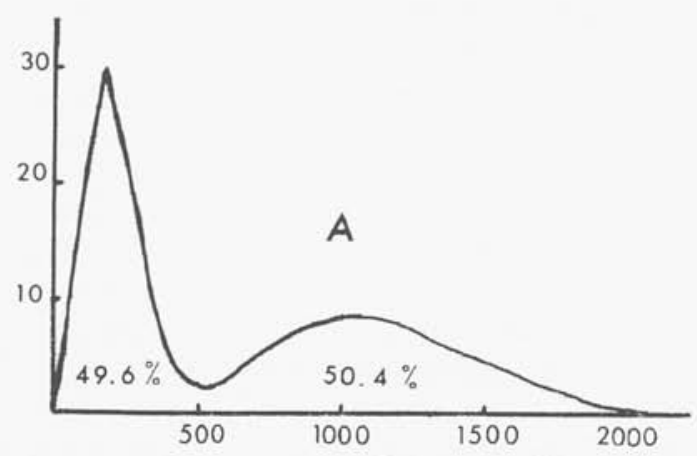

Fig. 1. - Evolution des coefficients de sédimentation des deux fractions giycogéniques du muscle d'Ascaris :

A : avant le jeûne ; 


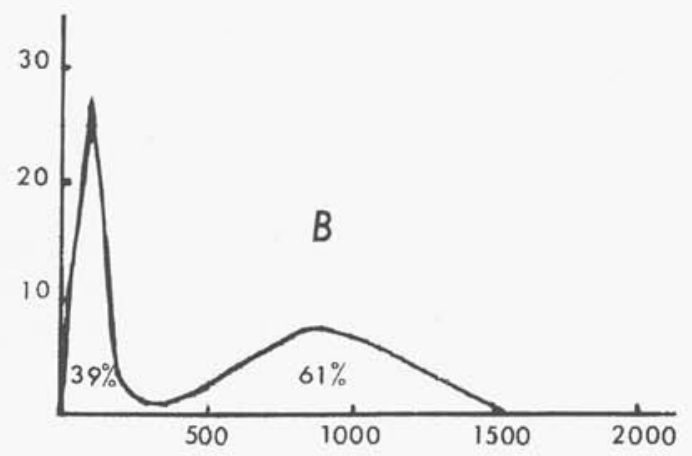

B : au bout de 24 heures de jeûne;

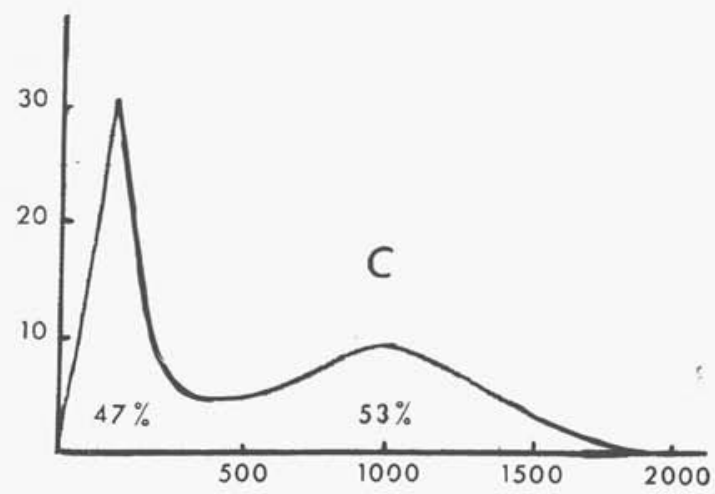

C: au bout de 24 heures de renutrition glucosée.

vie glucosé à $5 \%$. En regard sont consignés les caractéristiques de sédimentation des deux fractions, légère et lourde, du glycogène.

On constate que la diminution progressive de la concentration du muscle en glycogène s'accompagne d'une diminution du coefficient de sédimentation, donc du poids moléculaire moyen de chacune des deux fractions; en revanche, la restauration de la teneur en glycogène, au cours de la période de renutrition, permet, au bout de $24 \mathrm{~h}$, à chacune de ces fractions de se rapprocher très sensiblement de leurs caractéristiques initiales. D'autre part, le jeûne provoque un déséquilibre très net dans la répartition des deux fractions au bénéfice de la plus lourde puisque, à partir d'une répartition initiale à parties égales du bloc glycogénique, nous constatons qu'au bout de 24 heures de jeûne, la fraction légère ne représente plus que $39 \%$ du glycogène total alors que la fraction lourde en constitue $61 \%$; en revanche, la renutrition glucosée, en même temps que se reconstitue le stock glycogénique, permet aux deux fractions de se répartir à nouveau selon des proportions très proches de celles de départ. 
Il semble donc qu'il existe une relation entre la concentration du muscle en glycogène, le poids moléculaire et la répartition de chacune des deux fractions du polysaccharide ; on est fondé à s'interroger sur la signification de ces phénomènes en ce qui concerne les différences de métabolisme qu'ils laissent supposer. Ces mêmes questions se sont posées à Orrell, Bueding et Colucci (7) qui ont fait des observations identiques, chez Hymenolepis diminuta. Ultérieurement, Colucci, Orrell, Saz et Bueding (2) ont pu compléter leurs investigations en étudiant la différence des pourcentages d'incorporation du glucose marqué au ${ }^{14} \mathrm{C}$ dans chacune des deux fractions glycogéniques du même Cestode. Ils ont ainsi fait les observations suivantes:

- après incubation d'Hymenolepis diminuta dans un milieu contenant du glucose marqué, dans des conditions d'équilibre glucidique, l'activité de la fraction lourde est plus forte que celle de la fraction légère ;

- en revanche, pendant le jeûne, en même temps que décroît la teneur en glycogène et au cours de la resynthèse du polysaccharide sous l'influence du glucose dont une partie est marquée au ${ }^{14} \mathrm{C}$, l'activité de la fraction lourde est nettement inférieure à celle de l'activité légère. Les auteurs en concluent que la fraction à faible poids moléculaire est à la fois la plus rapidement utilisée au cours du jeûne et la première restaurée dans la phase de renutrition.

Le mécanisme de cette différence de comportement n'a pas encore été élucidé. Sans doute tient-il, soit à des réactions différentes pour chacune des deux fractions ou, au contraire, à un système enzymatique commun à toutes deux mais dont l'affinité est différente pour chacune d'entre elles.

Quoi qu'il en soit, l'existence, dans le muscle d'Ascaris, de deux fractions glycogéniques dont le comportement est différent suivant l'état de nutrition du parasite permet de penser que la plus légère représente la réserve glucidique immédiatement mobilisable à des fins métaboliques, tandis que la plus lourde paraît beaucoup plus inerte bien qu'elle puisse, semble-t-il constituer, après épuisement de la première, une ultıme possibilité de survie pour l'animal soumis à un jeûne glucidique sévère.

\author{
Annexe \\ TECHNIQUE D'ISOLEMENT ET DE PURIFICATION DU GLYCOGÈNE \\ D'Ascaris lumbricoides
}

a) homogénéisation du tissu avec quatre volumes d'une solution tampon au glycocolle $0,2 \mathrm{M}(\mathrm{pH} 10,4)$ et de deux volumes de chloroforme, à la température de $2^{\circ} \mathrm{C}$ à $4^{\circ} \mathrm{C}$;

b) centrifugation à 1.000 tours par minute pendant dix minutes à $0^{\circ} \mathrm{C}$. On recueille le surnageant aqueux (surnageant I) ; 
c) l'interphase et la phase inférieure sont reprises, additionnées de deux volumes de la solution tampon au glycocolle, à $2-4^{\circ} \mathrm{C}$. Une nouvelle centrifugation, dans les mêmes conditions que la première, permet de recueillir un surnageant II ;

d) on répète une extraction identique à quatre autres reprises ;

e) tous les surnageants sont réunis et soumis à une centrifugation de 26.000 tours par minute pendant 16 heures à $0^{\circ} \mathrm{C}$;

f) le résidu est repris par un volume d'eau de façon à avoir une concentration en glycogène de 0,6 à $0,8 \%$. Cette solution est additionnée d'un demi-volume du mélange chloroforme-alcool octylique $(3 \mathrm{v} / 1 \mathrm{v})$ et agitée mécaniquement pendant six heures ;

g) le mélange est centrifugé à 1.000 tours par minute pendant cinq minutes. La phase aqueuse est recueillie ;

h) on répète le même traitement sur la phase aqueuse jusqu'à ce qu'aucun précipité n'apparaisse plus à l'interphase ;

i) on termine par la précipitation du glycogène dissous dans la phase aqueuse par addition de 1,1 volume d'alcool éthylique ;

j) ce glycogène est séparé par centrifugation et séché sous vide.

\section{Bibliographie}

1. CAVier (R.) et SAVel (J.), 1952. — La synthèse du glycogène à partir de quelques glucides et de certains de leurs dérivés par l'Ascaris du porc, Ascaris lumbricoides Linné, 1758 C.R. Acad. Sc., 234, 2562-2564.

2. Colucci (A. V.), Orrell (S. A.), Saz (H. J.) et Bueding (E.), 1966. - Differential glucose incorporation into glycogène by Hymenolepis diminuta. J. biol. Chem., 241, 464-468.

3. Fairbarn (D.) et Passey (R. F.), 1957. - The occurence and distribution of trehalose and glycogen in the eggs and tissues of Ascaris lumbricoides Experim. Parasitol., 6, 566574.

4. Harrap (B. S.) et Manners (D. J.), 1952. - Molecular weight of glycogen determined by a light-scattering method. Nature, 170, 419-420.

5. KJolberg (D.), Manners (D. J.) et Wright (A.), 1963. - $\propto 1,4$ glucosans XVII. - The molecular structure of some glycogens. Comp. Biochem. Physiol., 8, 353-365.

6. Orrell (S. A.) et Bueding (E.), 1958. - Sedimentation characteristics of glycogen. $J$. Amer. Chem. Soc., 80, 3800.

7. Orrell (S. A.), Bueding (E.) et Colucci (A. V.), 1966. - Relationship between sedimentation coefficient distribution and glycogen level in the cestode Hymenolepis diminuta. Comp. Biochem. Physiol., 18, 657-662.

8. Orrell (S. A.), Bueding (E.) et Reissig (M.), 1964. - Physical caracteristics of undegraded glycogen. In Control of glycogen metabolism. Ciba Foundation Symposium, 29-44. 